Pacific Journal of Mathematics

COHOMOLOGY OPERATIONS FROM HIGHER PRODUCTS IN

вонUмн CEN kL 


\title{
COHOMOLOGY OPERATIONS FROM HIGHER PRODUCTS IN THE DERHAM COMPLEX
}

\author{
Bohumil Cenkl
}

\begin{abstract}
We give a new construction of all Steenrood cyclic reduced powers $\mathscr{P}^{i}$ and of Pontrjagin-Thomas $p$ th powers $\mathscr{B}_{p}$ for each prime $p$. The cohomology operations are indued by operations, analogous to the $p$-fold cup- $i$ products, defined in the deRham complex of CartanMiller. These operations form a basis of all the cohomology operations derived from cyclic groups. This extends the construction of the Steenrod squares based on the analogue of the cup- $i$ product in the deRham complex. From the construction of these new operations in the deRham complex it follows that the commutative cochain problem does not have a solution over the integers.
\end{abstract}

A key feature of cohomology is the existence of a commutative multiplication. This multiplication is induced by the cup product on cochains. While the product gives the cohomology a structure of a commutative ring, the cup product on the cochains is not commutative for arbitrary coefficients. The large number of cochains on any given space together with non-commutativity of the cup product makes any effective computation with cochains difficult.

Motivated by the rational deRham complex and its success in the rational homotopy theory, attempts were made to construct, for any space, a cochain complex with a commutative multiplication whose cohomology would be the cohomology of the space for any coefficient ring. One such construction was given by Cartan and Miller ([1], [4]). The commutative product in their complex induces the multiplication on cohomology with integer coefficients up to an additional factor, depending on the representing cochains. Another commutative cochain complex was constructed by Cenkl and Porter ("DeRham theorem with cubical forms," Pacific J. Math. 112 (1984), 35-48). In that paper we used a ring system for coefficients to solve the commutative cochain problem (as formulated in $\S 5$ ) for any space in terms of polynomial differential forms on simplices. The solution is the best possible in the sense of Theorem 2 . The multiplication induced on cohomology by the commutative product of forms in that complex is exactly the usual one. A construction of the cohomology operations 
could be also done by defining an appropriate generalized product in that complex.

The deRham complexes of Cartan-Miller and Cenkl-Porter have been used for the computations of the cohomology of the fundamental groups of nilmanifolds. The deRham complex of Cenkl-Porter also turned out to be suitable for the construction of a free model in the tame homotopy theory.

The constructions of the cohomology operations presented in this paper constitute part of the program to study the role of commutative algebras in homotopy theory. In fact it seems to be the first construction of the cohomology operations based on the operations in a commutative cochain algebra.

1. Products of cochains, $\mathscr{P}^{i}$ and $\mathscr{B}_{p}$. Let $R$ be a commutative ring with a unit and $\mathscr{S}$ the category of simplicial sets. Denote by $\mathscr{M}_{R}, d \mathscr{M}_{R}^{*}$ and $d \mathscr{M}_{R}^{*, *}$ the category of $R$-modules, differential graded $R$-modules and differential bigraded $R$-modules respectively.

Denote by $A=A^{*, *}=A_{R}^{*, *}: \mathscr{S} \rightarrow d \mathscr{M}_{R}^{*, *}$ the deRham functor of Cartan and Miller ([1], [4]), and by $\Gamma_{R}=\Gamma_{R}(t)$ the divided power algebra over $R$ on a single generator $t . \quad \Gamma_{R}=\bigoplus_{q \geq 0} \Gamma_{q}$, where $\Gamma_{q}$ denotes the free $R$-modules on a single generator $\gamma_{q}=\gamma_{q}(t) . \Gamma_{R}$ is given the structure of a commutative ring with unit $\gamma_{0}=1$ by the pairing

$$
\gamma: \Gamma_{p} \otimes \Gamma_{q} \rightarrow \Gamma_{p+q}
$$

defined by

$$
\gamma_{p} \gamma_{q}=\frac{(p+q) !}{p ! q !} \gamma_{p+q}
$$

In general, the associativity of this pairing gives the map

$$
\begin{gathered}
\gamma: \Gamma_{q_{1}} \otimes \cdots \otimes \Gamma_{q_{k}} \rightarrow \Gamma_{q_{1}+\cdots+q_{k}}, \\
\gamma_{q_{1}} \cdots \gamma_{q_{k}}=\frac{\left(q_{1}+\cdots+q_{k}\right) !}{q_{1} ! \cdots q_{k} !} \gamma_{q_{1}+\cdots+q_{k}} .
\end{gathered}
$$

Denote by $C_{*}: \mathscr{S} \rightarrow d \mathscr{M}_{R}^{*}$ the functor $C_{p}(X)=$ the free $R$-module of $p$-chains generated by the $p$-simplices. If $p<0, C_{p}(X)=0$ for any $X \in \mathscr{S}$. The boundary operator is denoted by $\partial: C_{p} \rightarrow C_{p-1}$. Let $C=$ $C^{*, *}: \mathscr{S} \rightarrow d \mathscr{M}_{R}^{*, *}$ be the functor defined by $C^{p, q}(X)=C^{p}\left(X ; \Gamma_{q}\right)=$ the normalized $p$-cochains with coefficients in $\Gamma_{q}$. If $p<0$ or $q<0$ then $C^{p, q}(X)=0$ for any $X \in \mathscr{S}$. The coboundary operator is denoted by $d: C^{p, q} \rightarrow C^{p+1, q}$. The index, $\operatorname{In}(c)$, of a 0 -chain $c \in C_{0}(X)$ is the sum of its coefficients. 
In his original construction of the reduced powers $\mathscr{P}^{i}$ in [5] Steenrod uses a family of chain operations. Let us recall the relevant points of Steenrod's construction.

Let $\pi$ be the cyclic permutation group of degree $p$ and order $p$ for any integer $p$ and $\mathbb{Z}[\pi]$ be the group ring. Denote by $s: \mathbb{Z}[\pi] \rightarrow \mathbb{Z}$ the homomorphism $s\left(\sum x_{i} g_{i}\right)=\sum x_{i}, x_{i} \in \mathbb{Z}, g_{i} \in \pi$. An infinite sequence $\alpha_{1}, \alpha_{2}, \ldots$, of elements of $\mathbb{Z}[\pi]$ is called the 0 -sequence if $s\left(\alpha_{1}\right)=0$, and $\alpha_{i+1} \alpha_{i}=0$ for $i=1,2, \ldots$ Denote by $T$ the generator of $\pi$,

$$
T\left(a_{1}, a_{2}, \ldots, a_{p}\right)=\left(a_{p}, a_{1}, a_{2}, \ldots, a_{p-1}\right),
$$

where $a_{1}, \ldots, a_{p}$ is a set of $p$ letters, and by $I$ the identity permutation. Then the elements of $\mathbb{Z}[\pi]$

$$
\begin{aligned}
& \alpha_{2 j-1}=T-I, \\
& \alpha_{2 j}=I+T+T^{2}+\cdots+T^{p-1},
\end{aligned}
$$

$j=1,2, \ldots$, form a 0 -sequence. From [5], Lemma 5.5, it follows that there exists a sequence of homomorphisms

$$
\begin{aligned}
D_{i}: C_{n}(X) \rightarrow[\underbrace{C_{*}(X) \otimes \cdots \otimes C_{*}(X)}_{p \text {-times }}]_{n+i} \\
=\bigoplus_{\substack{n_{1}+\cdots+n_{p}=n+i \\
n_{j} \geq 0}}\left\{C_{n_{1}}(X) \otimes \cdots \otimes C_{n_{p}}(X)\right\},
\end{aligned}
$$

$i=0,1,2, \ldots$, such that:

(i) $\operatorname{In}\left(D_{0} C\right)=\operatorname{In}(c)$ for any $c \in C_{0}(X)$,

(ii) $\partial D_{i}+(-1)^{i+1} D_{i} \partial-\alpha_{i} D_{i-1}, i=1,2, \ldots$

Definition. The $p$-fold cup- $i$ product

$$
\begin{gathered}
\bigcup_{i}^{p}=\phi_{\cup_{i}}^{p}: C^{n_{1}, q_{1}}(X) \otimes \cdots \otimes C^{n_{p}, q_{p}}(X) \rightarrow C^{n-i, q}(X), \\
n=n_{1}+\cdots+n_{p}, \quad q=q_{1}+\cdots+q_{p},
\end{gathered}
$$

is the composition of the map

$$
\begin{gathered}
\psi_{i}^{p}: C^{n_{1}, q_{1}}(X) \otimes \cdots \otimes C^{n_{p}, q_{p}}(X) \rightarrow C^{n-i}\left(X ; \Gamma_{q_{1}} \otimes \cdots \otimes \Gamma_{q_{p}}\right), \\
\psi_{i}^{p}\left(c_{1} \otimes \cdots \otimes c_{p}\right)(\sigma)=\left(c_{1} \otimes \cdots \otimes c_{p}\right) D_{i}(\sigma)
\end{gathered}
$$

with the $p$-bold product $\gamma: \Gamma_{q_{1}} \otimes \cdots \otimes \Gamma_{q_{p}} \rightarrow \Gamma_{q}$; i.e.,

$$
\phi_{\cup_{i}}^{p}=\gamma \circ \psi_{i}^{p} \text {. }
$$

$\bigcup_{i}^{p}=\phi_{\cup_{i}}^{p}=0$ in dimensions $<i$. 
From the identity (ii) it follows that

$$
d \phi_{\cup_{i}}^{p}+(-1)^{i+1} \phi_{\cup_{i}}^{p} d=(-1)^{i+1} \phi_{\cup_{i-1}}^{p} \circ \alpha_{i},
$$

$i=1,2, \ldots, p \geq 2$.

In particular $\phi_{\cup_{i}}^{2}=\phi_{\cup_{i}}=\bigcup_{i}$ is the cup- $i$ product and $\phi_{\cup_{0}}^{p}$ is the $p$-fold cup product.

Cohomology operations. Here we assume that $p$ is a prime. Let $e_{q}: C^{*}\left(X ; \Gamma_{q}\right) \rightarrow C^{*}\left(X ; \mathbb{Z}_{p}\right)$ be the map induced by the coefficient map which sends $\gamma_{q}$ to the generator 1 of $\mathbb{Z}_{p}$. Define

$$
\begin{gathered}
\tilde{\phi}_{i}^{p}: C^{n_{1}}\left(X ; \mathbb{Z}_{p}\right) \otimes \cdots \otimes C^{n_{p}}\left(X ; \mathbb{Z}_{p}\right) \rightarrow C^{n-i}\left(X ; \mathbb{Z}_{p}\right), \\
\frac{\left(q_{1}+\cdots+q_{p}\right) !}{q_{1} ! \cdots q_{p} !} \tilde{\phi}_{i}^{p} \circ\left(c_{q_{1}} \otimes \cdots \otimes e_{q_{p}}\right)=e_{q} \circ \phi_{\cup_{i}}^{p} .
\end{gathered}
$$

Let $\hat{\phi}_{i}^{p}$ be the map induced by $\tilde{\phi}_{i}^{p}$ on the cohomology restricted to the diagonal. Then the $p$ th cyclic reduced power, $\mathscr{P} i$, of Steenrod [7] is the operation

$$
\mathscr{P}^{i}: H^{n}\left(X ; \mathbb{Z}_{p}\right) \rightarrow H^{n+2 i(p-1)}\left(X ; \mathbb{Z}_{p}\right), \quad i=0,1, \ldots .
$$

For any $u \in H^{n}\left(X ; \mathbb{Z}_{p}\right)$

$$
\begin{aligned}
\mathscr{P}^{i} u & =(-1)^{m i+n m(n-1) / 2}(m !)^{2 i-n} \hat{\phi}_{i}^{p}\left(u^{p}\right), \quad m=\frac{1}{2}(p-1), \\
u^{p} & =u \otimes \cdots \otimes u(p \text {-times }) .
\end{aligned}
$$

Let $e_{q}^{j}: C^{*}\left(X ; \Gamma_{q}\right) \rightarrow C^{*}\left(X ; \mathbb{Z} / p^{j} \mathbb{Z}\right)$ be the map induced by the coefficient $\operatorname{map} \Gamma_{q} \rightarrow \mathbb{Z} / p^{j} \mathbb{Z}, \gamma_{q} \rightarrow 1$. Furthermore, let $\Sigma^{*}=\sum_{k=1}^{p-1} k T^{p-k} \in$ $\mathbb{Z}[\pi]$. Then we define the map

$$
\begin{gathered}
\tilde{\phi}^{p}: C^{n_{1}}\left(X ; \mathbb{Z} / p^{k} \mathbb{Z}\right) \otimes \cdots \otimes C^{n_{p}}\left(X ; \mathbb{Z} / p^{k} \mathbb{Z}\right) \rightarrow C^{p n}\left(X ; \mathbb{Z} / p^{k+1} \mathbb{Z}\right), \\
n=n_{1}+\cdots+n_{p} \text {, by } \\
\frac{\left(q_{1}+\cdots+q_{p}\right) !}{q_{1} ! \cdots q_{p} !} \tilde{\phi}^{p}\left(e_{q_{1}}^{k} \otimes \cdots \otimes e_{q_{p}}^{k}\right) \\
=e_{q}^{k+1} \circ \gamma \circ\left\{\psi_{0}^{p}+(-1)^{n^{2}-p} \psi_{1}^{p} d \Sigma^{*}\right\} .
\end{gathered}
$$

If $\hat{\phi}^{p}$ is the restriction to the diagonal of the map induced by $\tilde{\phi}^{p}$ on the cohomology then the Pontrjagin-Thomas $p$ th power is the operation

$$
\mathscr{B}_{p}: H^{2 m}\left(X ; \mathbb{Z}_{p^{k}}\right) \rightarrow H^{2 m p}\left(X ; \mathbb{Z}_{p^{k+1}}\right), \quad n=2 m,
$$

such that

$$
\mathscr{B}_{p} u=\hat{\phi}^{p}\left(u^{p}\right), \quad u^{p}=u \otimes \cdots \otimes u \text { (p-times). }
$$


2. Main results. Let $A: \mathscr{S} \rightarrow d \mathscr{M}_{R}^{*, *}$ be the deRham functor of Cartan-Miller ([1],[4]), and let

$$
\mu: A \rightarrow C
$$

be the linear transformation induced by the integration of forms over simplices. This map induces isomorphism of modules

$$
H^{p}\left(A^{*, q}(X)\right) \stackrel{\mu^{*}}{\longrightarrow} H^{p}\left(X ; \Gamma_{q}\right)
$$

which commutes with the wedge and cup products.

There exists a family of cochain operations on the deRham complex $A(X)$ inducing the Steenrod cyclic reduced powers and the PontrjaginThomas $p$ th powers for each prime $p$. The following properties of the cup and wedge products and of the map $\mu$ allow us to define a family of such operations:

(i) The wedge product on $A(X)$ is commutative and associative.

(ii) The cup product on $C(X)$ is associative and commutative in dimension zero.

(iii) The map $\mu$ is multiplicative in dimension zero.

(iv) There exists a transformation of functors $\tau: C \rightarrow A$ which is a chain homotopy equivalence such that $\mu \tau=$ identity.

The proof of (i) and (iii) can be found in [1] and [4]; (ii) is a standard fact; (iv) is proved by acyclic model argument.

THEOREM 1. There are natural maps $\phi_{i}^{p}=\stackrel{i}{\wedge}_{p}$, called $p$-fold wedge- $i$ products, such that the diagram

$$
\begin{aligned}
& A_{R}^{n_{1}, q_{1}}(X) \otimes \cdots \otimes A_{R}^{n_{p}, q_{p}}(X) \stackrel{\phi_{i}^{p}}{\longrightarrow} A_{R}^{n-i, q}(X) \\
& \mu \otimes \cdots \otimes \mu \downarrow \\
& C^{n_{1}}\left(X ; \Gamma_{q_{1}}\right) \otimes \cdots \otimes C^{n_{p}}\left(X ; \Gamma_{q_{p}}\right) \stackrel{\phi_{\mathrm{u}_{i}}^{p}}{\longrightarrow} C^{n-i}\left(X ; \Gamma_{q}\right),
\end{aligned}
$$

$n=n_{1}+\cdots+n_{p}, q=q_{1}+\cdots+q_{p}$, is homotopy commutative. The $p$-fold cup-i product $\phi_{\cup_{i}}^{p}$ is defined via the cochain operations of Steenrod [5].

Corollary 1. Let $a_{j} \in A_{\mathbb{Z}_{p}}^{n_{j}, q_{j}}(X), j=1,2, \ldots, p$, be cocycles such that $e_{q_{i}} \mu\left(a_{i}\right)=e_{q_{j}} \mu\left(a_{j}\right)$ in $C^{*}\left(X ; \mathbb{Z}_{p}\right)$, for all $i, j=1,2, \ldots, p$. Then, for $i \leq p, \phi_{i}^{p}\left(a_{1}^{p}\right), a_{1}^{p}=a_{1} \otimes \cdots \otimes a_{1}$ (p-times) is a cocycle in $A_{\mathbb{Z}_{p}}^{n p-i, q}(X)$, 
$n=n_{1}=\cdots=n_{p}, q=q_{1}+\cdots+q_{p}$, whose class is related to the cyclic reduced power of Steenrod

$$
\mathscr{P}^{i}: H^{n}\left(X ; \mathbb{Z}_{p}\right) \rightarrow H^{n+2 i(p-1)}\left(X ; \mathbb{Z}_{p}\right)
$$

by the following formula:

$$
\begin{aligned}
& \frac{\left(q_{1}+\cdots+q_{n}\right) !}{q_{1} ! \cdots q_{n} !} \mathscr{P}^{i}\left[e_{q_{1}}\left(\mu\left(a_{1}\right)\right)\right] \\
& \quad=(-1)^{m i+m n(n-1) / 2}(m !)^{2 i-n}\left[\phi_{i}^{p}\left(a_{1}^{p}\right)\right], \quad m=(p-1) / 2 .
\end{aligned}
$$

[*] stands for the cohomology class determined by the cocycle *.

CoRollary 2. Let $a_{j} \in A_{\mathbb{Z}_{p^{k}}}^{n, a_{j}}(X), j=1,2, \ldots, p$ be cocycles such that $e_{q_{i}} \mu\left(a_{i}\right)=e_{q_{j}} \mu\left(a_{j}\right)$ in $C^{*}\left(X ; \mathbb{Z}_{p^{k}}\right)$ for $i, j=1,2, \ldots, p$. Then

$$
\mathscr{B}_{p}\left(a_{1}\right)=\left(\phi_{0}^{p}+(-1)^{n^{2}-p} \phi_{1}^{p} d \Sigma^{*}\right)\left(a_{1}^{p}\right)
$$

is a cocycle in $A_{\mathbb{Z}_{p} k+1}^{n p}(X)$ and

$$
\frac{\left(q_{1}+\cdots+q_{p}\right) !}{q_{1} ! \cdots q_{p} !} \mathscr{B}_{p}\left[a_{q_{1}} \mu\left(a_{1}\right)\right]=\left[\mathscr{B}_{p}\left(a_{1}\right)\right]
$$

CoRollaRy 3 ([2]). In particular the Steenrod squares are constructed from the 2-fold wedge-i products, called simply wedge-i products and denoted by $\stackrel{i}{\wedge}$, as follows:

Let $a_{1}, a_{2}$ be cocycles in $A_{\mathbb{Z}_{2}}^{p, q_{1}}(X), A_{\mathbb{Z}_{2}}^{p, q_{2}}(X)$ respectively with $e_{q_{1}} \mu\left(a_{1}\right)$ $=e_{q_{2}} \mu\left(a_{2}\right)$ in $C^{p}\left(X ; \mathbb{Z}_{2}\right)$. For $i \leq p, a_{1} \stackrel{i}{\wedge} a_{2}$ is a coocyle in $A_{\mathbb{Z}_{2}}^{2 p-i, q_{1}+q_{2}}(X)$ whose class is related to the Steenrod squares by the identity

$$
\left[e_{q_{1}+q_{2}} \mu\left(a_{1} \stackrel{i}{\wedge} a_{2}\right)\right]=\frac{\left(q_{1}+q_{2}\right) !}{\left(q_{1}\right) !\left(q_{2}\right) !} S_{q}^{p-1}\left[\mu\left(a_{1}\right)\right] .
$$

3. $p$-fold wedge- $i$ product on forms. In this part we use the following notation

$$
\begin{aligned}
A_{i} & =A \otimes \cdots \otimes A \quad(i \text {-times }), \\
A_{i}^{k, q} & =\bigoplus_{\substack{k_{1}+\cdots+k_{i}=k \\
q_{1}+\cdots q_{i}=q}}\left(A^{k_{1}, q_{1}} \otimes \cdots \otimes A^{k_{i}, q_{i}}\right), \\
A_{i}^{k} & =A_{i}^{k, *}
\end{aligned}
$$


and similarly

$$
C_{i}=C \otimes \cdots \otimes C \quad(i \text {-times }), \text { etc. }
$$

The functors $A, C$ and the transformation

$$
\mu: A \rightarrow C
$$

have the following important properties: The functors $A_{i}^{k, q}$ are acyclic on models in the dimensions bounded by the filtration $q$ from above ([2], [3]); $C^{k, q}$ is corepresentable; $\mu$ commutes with products in dimension zero. Furthermore, both the wedge product on $A$ and the cup product on $C$ are associative. Therefore the $p$-fold wedge product

$$
\phi_{\wedge}^{p}: A_{p} \rightarrow A
$$

and the $p$-fold cup product

$$
\phi_{\cup}^{p}: C_{p} \rightarrow C
$$

are well-defined.

Hence we can prove, by the method of acyclic models that:

PROPOSITION. There exist transformations of functors

$$
\begin{gathered}
\mu_{p, 1}=\left\{\mu_{p, 1}^{k}\right\}, \\
\mu_{p, 1}^{k}: A^{k_{1}, q_{1}} \otimes \cdots \otimes A^{k_{p}, q_{p}} \rightarrow C^{k-1, q}, \\
k \geq 1, n \geq 2, k=k_{1}+\cdots+k_{p}, q=q_{1}+\cdots+q_{p},
\end{gathered}
$$

such that

$$
d \mu_{p, 1}^{k-1}+\mu_{p, 1}^{k} d=\mu \phi_{\wedge}^{p}=\phi_{\cup}^{p}\left(\mu^{p}\right), \quad \mu^{p}=\mu \otimes \cdots \otimes \mu(p \text {-times }),
$$

where $\mu_{p, 1}^{0}=0$.

REMARK. $\mu_{p, 1}$ is a generalization of the transformation $\mu_{2,1}$ used by Gugenheim [3] to prove that the map of the bar constructions $B(\mu): B(A) \rightarrow B(C)$, induced by $\mu$, is a map of coalgebras.

The transformation $\mu_{2,1}$ of [3] extends also in another direction.

Lemma. Let $\mu: A \rightarrow C$ be the transformation of functors induced by integration and let

$$
\begin{aligned}
\phi_{\cup_{i}}^{p}: C^{k_{1}, q_{1}} \otimes \cdots \otimes C^{k_{p}, q_{p}} \rightarrow C^{k-i, q}, & \\
& k=k_{1}+\cdots+k_{p}, \quad q=q_{1}+\cdots+q_{p},
\end{aligned}
$$


be the p-fold cup-i product and let

$$
\begin{aligned}
\phi_{\wedge}^{p}: A^{k_{1}, q_{1}} \otimes \cdots \otimes A^{k_{p}, q_{p}} \rightarrow A^{k, q}, & \\
& k=k_{1}+\cdots+k_{p}, \quad q=q_{1}+\cdots+q_{p},
\end{aligned}
$$

be the p-fold wedge product.

Then there exist natural transformations

$$
\begin{gathered}
\mu_{p, i}=\left\{\mu_{p, i}^{k}\right\}, \\
\mu_{p, i}^{k}: A^{k_{1}, q_{1}} \otimes \cdots \otimes A^{k_{p}, q_{p}} \rightarrow C^{k-i, q}, \quad i \geq 1,
\end{gathered}
$$

such that

$$
\begin{array}{r}
d \mu_{p, i}^{k-1}+(-1)^{i+1} \mu_{p, i}^{k} d=(-1)^{i+1} \mu_{p, i-1}^{k-1} \circ \alpha^{i-1}-\phi_{u_{i-1}}^{p}\left(\mu^{p}\right), \\
\mu^{p}=\mu \otimes \cdots \otimes \mu(p \text {-times }),
\end{array}
$$

for $i \geq 2$, where the existence and properties of the transformations $\mu_{p, 1}^{k}$ are given by the Proposition.

Suppose that $\alpha_{1}, \alpha_{2}, \ldots$ is the 0 -sequence used in the definition of the $p$-fold cup- $i$ product and that $\tau: C^{k, q} \rightarrow A^{k, q}$ is a transformation of functors with the properties: $d \tau=\tau d, \mu \tau=I$, and that $\tau \mu$ is chain homotopic to the identity ([2]).

DEFINITION. The $p$-fold wedge- $i$ product $\left(\phi_{i}^{p}\right.$-product) is the map

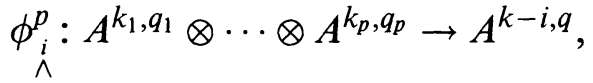

$$
\begin{aligned}
& k=k_{1}+\cdots+k_{p}, \quad q=q_{1}+\cdots+q_{p}, \\
& \phi_{i}^{p}=(-1)^{i} \tau \circ \mu_{p, i} \circ \alpha_{i} \text { for } i \geq 1 \text {, } \\
& \phi_{0}^{p}=\phi_{\wedge}^{p}=\text { the } p \text {-fold wedge product. }
\end{aligned}
$$

\section{Proofs.}

Proof of the Proposition. The statement is proved by the method of acyclic models. Denote by $\hat{A}_{i}^{k}, \hat{C}_{i}$ the functors associated with $A_{i}^{k}$, $C_{i}$ respectively and by $\phi: A_{i}^{k} \rightarrow \hat{A}_{i}^{k}, \phi: C_{i} \rightarrow \hat{C}_{i}$ the transformations which composed with the canonical maps $\psi: \hat{A}_{i}^{k} \rightarrow A_{i}^{k}, \psi: \hat{C}_{i} \rightarrow C_{i}$ give the identity. See [2] or [3] for the definitions. 
Suppose that $p$ is an integer $p \geq 2$. Consider the diagram

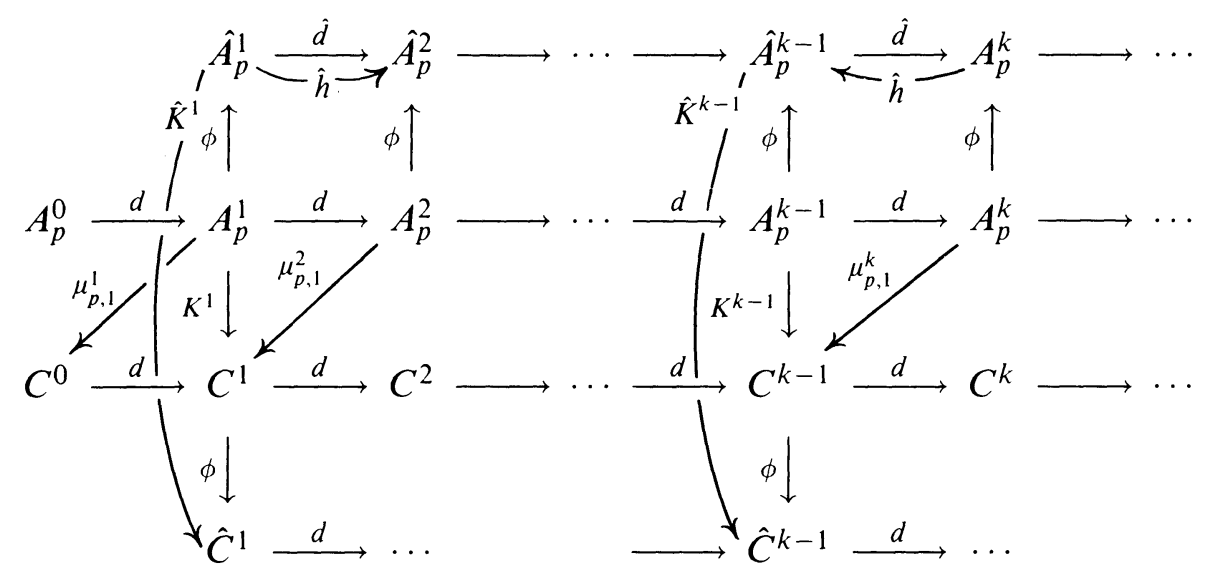

where $\hat{h}$ is the chain homotopy whose existence follows from the acyclicity of $A_{p}^{k}$ on models.

Let $K=\mu \phi_{\wedge}^{p}-\phi_{\cup}^{p}\left(\mu^{p}\right), \mu^{p}=\mu \otimes \cdots \otimes \mu$ (p-times), $K^{k}=K \mid A_{p}^{k}$ for $k \geq 1, K^{0}=0$. Define $\mu_{p, 1}=\left\{\mu_{p, 1}^{k}\right\}$ inductively by $k$. Set $\mu_{p, 1}^{0}=0$ and define $\mu_{p, 1}^{1}$ so that $\mu_{p, 1}^{1} d=0$ and then extend arbitrarily to $A_{p}^{1}$. Since $K^{0}=0$ we have $\mu_{p, 2}^{1} d=K^{0}$ on the elements of degree zero. Define

$$
\mu_{p, 1}^{2}=\psi \hat{K}^{1} \hat{h} \phi-d \mu_{p, 1}^{1} \text {. }
$$

Since $K^{1} d=0$ on the elements of degree zero we get

$$
\mu_{p, 1}^{2} d=K^{1},
$$

or equivalently

$$
d \mu_{p, 1}^{1}+\mu_{p, 1}^{2} d=\mu \phi_{\wedge}^{p}-\phi_{\cup}^{p}\left(\mu^{p}\right) .
$$

Inductively, assume that $\mu_{p, 1}^{l}$ has been defined and satisfies the identities

$$
d \mu_{p, 1}^{l-1}+\mu_{p, 1}^{l} d=\mu \phi_{\wedge}^{p}-\phi_{\cup}^{p}\left(\mu^{p}\right) \quad \text { for } l \leq k-1 .
$$

Then for

$$
\begin{aligned}
W^{l} & =K^{l}-d \mu_{p, 1}^{l} \\
W^{l} d & =0 \quad \text { for } l \leq k-1 .
\end{aligned}
$$

If we define

$$
\mu_{p, 1}^{k}=\psi \hat{W}^{k-1} \hat{h} \phi
$$


then

$$
\mu_{p, 1}^{k} d=W^{k-1}
$$

which completes the proof.

Proof of the Lemma. We assume that the formula

$$
\begin{aligned}
d \mu_{p, j}^{l-1}+(-1)^{j+1} \mu_{p, j}^{l} d & \\
=(-1)^{j+1} \mu_{p, j-1}^{l-1} \circ \alpha_{j-1}-\phi_{\cup_{j-1}}^{p}\left(\mu^{p}\right), \mu^{p}=\mu \otimes & \cdots \otimes \mu \\
\quad & \quad(p \text {-times), }
\end{aligned}
$$

has been proved for $l=1,2, \ldots$ and $j \leq i-1$. We want to show that it is valid also for $j=i$ and for $l=1,2, \ldots$.

Define $\mu_{p, i}^{k}=0$ for $k=0,1, \ldots, i-1$. Consider the diagram

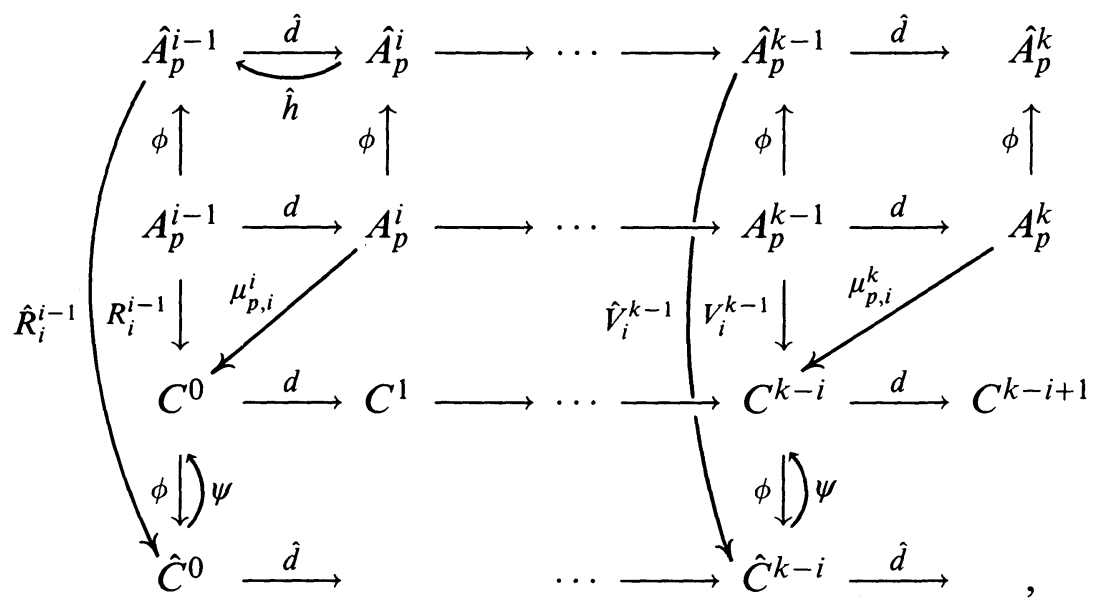

where $R_{i}^{i-1}$ is defined by

$$
R_{i}^{i-1}=(-1)^{i+1} \mu_{p, i-1}^{i-1} \circ \alpha_{i-1}-\phi_{\cup_{i-1}^{p}}^{p}\left(\mu^{p}\right) .
$$

Since $\alpha_{i-2} \circ \alpha_{i-1}=0, \phi_{\cup_{i-1}}^{p} d=(-1)^{i+1} d \phi_{\cup_{i-1}}^{p}+\phi_{\cup_{i-2}}^{p} \alpha_{i-1}$ and $\phi_{\cup_{i-1}}^{p}\left(\mu^{p}\right)$ is zero on $A_{p}^{i-2}$ we get

$$
R_{i}^{i-1} d=0
$$

Define $\mu_{p, i}^{i}$ by

$$
\mu_{p, i}^{i}=(-1)^{i+1} \psi \hat{R}_{i}^{i-1} \hat{h} \phi .
$$

Then

$$
(-1)^{i+1} \mu_{p, i}^{i} d=R_{i}^{i-1} .
$$


Now we assume that the formula (1) is proved for $l<k$ and $j \leq i$. Set

$$
V_{i}^{k-1}=(-1)^{i+1} \mu_{p, i-1}^{k-1} \circ \alpha_{i-1}-\phi_{\cup_{i-1}}^{p}\left(\mu^{p}\right)-d \mu_{p, i}^{k-1} .
$$

Then

$$
\begin{aligned}
V_{i}^{k-1} d= & (-1)^{i+1} \mu_{p, i-1}^{k-1} d \alpha_{i-1}-d \mu_{p, i-1}^{k-2} \alpha_{i-1} \\
& +(-1)^{i+1} d \phi_{\cup_{i-1}^{p}}^{p}\left(\mu^{p}\right)-\phi_{\cup_{i-1}^{p}}^{p} d\left(\mu^{p}\right) \\
= & (-1)^{i+1} \mu_{p, i-2}^{k-2} \alpha_{i-2} \circ \alpha_{i-1}=0 .
\end{aligned}
$$

Hence we define $\mu_{p, i}^{k}$ by

$$
\mu_{p, 2}^{k}=(-1)^{i+1} \psi \hat{V}_{i}^{k-1} \hat{h} \phi .
$$

From here we conclude that

$$
(-1)^{i+1} \mu_{p, i}^{k} d=V_{i}^{k-1},
$$

which completes the proof.

5. Nonexistence of commutative cochains over the integers. Let $R$ be a commutative ring.

We say that

$$
B^{*}: \mathscr{S} \rightarrow d \mathscr{M}_{R}^{*}
$$

solves the commutative cochain problem over $R$ if:

(i) $B^{*}$ is acyclic on models with unit

$$
\eta: R \rightarrow B^{*}
$$

(ii) there is a commutative transformation

$$
\Lambda: B^{*} \otimes B^{*} \rightarrow B^{*}
$$

with the diagram

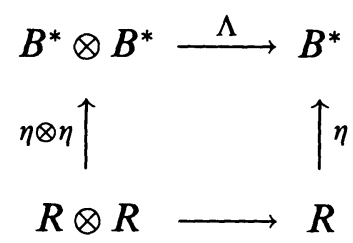

commutative;

(iii) there is a transformation $\mu: B^{*} \rightarrow C^{*}$ inducing an isomorphism

$$
\mu: H^{p}(B(X)) \rightarrow H^{p}(X ; R)
$$

for all $p$ and all simplicial sets $X$. 
THEOREM 2. Let $R$ be a commutative ring. If the commutative cochain problem over $R$ has a solution then for each element $a \in R$, the element $a^{2}$ in $R$ is divisible by two.

COROLlARY. There is no solution of the commutative cochain problem over the integers.

These results were obtained jointly with $\mathrm{R}$. Porter. They were announced in [2].

Proof of the Theorem. Assume that $B^{*}: \mathscr{S} \rightarrow d \mathscr{M}_{R}^{*}$ is acyclic on models with units $\eta_{B}: R \rightarrow B^{0}$, where $R$ is a commutative ring. Further assume that there is a commutative transformation $\Lambda: B^{*} \otimes B^{*} \rightarrow B^{*}$ with the diagram in (ii) commutative. Denote by $C^{*}: \mathscr{S} \rightarrow d \mathscr{M}_{R}^{*}$ the normalized cochains with $R$-coefficients.

From the acyclic model theorem it follows that there is a transformation $\mu: B^{*} \rightarrow C^{*}$, unique up to homotopy, with

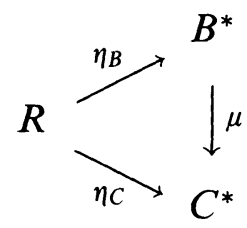

commutative. We assume that $\mu^{*}$ in (iii) is an isomorphism for all $p$ and all simplicial sets $X$.

Since $B^{*}$ is acyclic on models and $\wedge$ is commutative, the arguments used in the construction of higher homotopies applied to $B^{*}$ imply the existence of maps

$$
\mu_{i}:(B \otimes B)^{n} \rightarrow C^{n-i}
$$

such that

$$
d \mu_{1}+\mu_{1} d=\mu \phi_{\wedge}-\phi_{\cup}(\mu \otimes \mu)
$$

and

$$
d \mu_{i}+(-1)^{i+1} \mu_{i} d=\mu_{i-1}\left(T+(-1)^{i+1} I\right)-\phi_{\cup_{i-1}}(\mu \otimes \mu) \quad \text { for } i \geq 2 .
$$

In particular there are maps $\mu_{1}$ and $\mu_{2}$ with

$$
d \mu_{2}-\mu_{2} d=\mu_{1}(T-I)-\phi_{\cup_{1}}(\mu \otimes \mu) .
$$

Let $a \in R$. We want to show that $a^{2}$ is divisible by 2 . Choose a cocycle $\alpha \in B^{1}\left(S^{1}\right)$ such that the element $[\mu(\alpha)] \in H^{1}\left(S^{1} ; R\right) \simeq$ $\operatorname{Hom}_{\mathbb{Z}}(\mathbb{Z}, R)$ is the homomorphism which sends $1 \in \mathbb{Z}$ to the element $a$ in $R$. (Here $S^{1}$ denotes the circle.) 
From $(*)$ we have

$$
d \mu_{2}(\alpha \otimes \alpha)=-2 \mu_{1}(\alpha \otimes \alpha)-\mu(\alpha) \cup_{1} \mu(\alpha) ;
$$

hence $\mu(\alpha) \cup_{1} \mu(\alpha)$ represents the zero class in $H^{1}\left(S^{1} ; R / 2 R\right) \simeq R / 2 R$.

On the other hand a direct computation, using Steenrod definition of $\cup_{1}$, shows that the class $\left[\mu(\alpha) \cup_{1} \mu(\alpha)\right]$ in $H^{1}\left(S^{1} ; R / 2 R\right) \simeq$ $\operatorname{Hom}_{\mathbb{Z}}(\mathbb{Z}, R / 2 R)$ is the element which sends $1 \in \mathbb{Z}$ to the element $a^{2}$ in $R / 2 R$. Hence $a^{2}=0$ in $R / 2 R$.

\section{REFERENCES}

[1] H. Cartan, Théorie cohomologiques, Invent. Math., 35 (1976), 261-271.

[2] B. Cenkl, and R. Porter, Cup-i product and higher homotopies in the deRham complex, Publications Secció de Matematiques, Univ. Barcelona, 26 (1982), 9-29.

[3] V. K. A. M. Gugenheim, On the multiplicative structure of the deRham theory, J. Diff. Geom., 11 (1976), 309-314.

[4] E. Y. Miller, DeRham cohomology with arbitrary coefficients, Topology, 17 (1978), 193-203.

[5] N. E. Steenrod, Reduced powers of cohomology classes, Ann. of Math., 56 (1952), 47-67.

[6] N. E. Steenrod, and D. B. A. Epstein, Cohomology operations, Annals of Mathematics Studies, 50 (1962), pp. 138.

[7] N. E. Steenrod, and E. Thomas, Cohomology operations derived from cyclic groups, Comment. Math. Helv., 32 (1957), 129-152.

Received September 30, 1987 and in revised from November 9, 1988.

NORTHEASTERN UNIVERSITY

Boston, MA 02115 



\section{PACIFIC JOURNAL OF MATHEMATICS EDITORS}

\author{
V. S. VARADARAJAN \\ (Managing Editor) \\ University of California \\ Los Angeles, CA 90024-1555-05 \\ Herbert Clemens \\ University of Utah \\ Salt Lake City, UT 84112 \\ THOMAS ENRIGHT \\ University of California, San Diego \\ La Jolla, CA 92093
}

R. FINN

Stanford University

Stanford, CA 94305

HeRMANN FlaschKa

University of Arizona

Tucson, AZ 85721

VAUGHaN F. R. Jones

University of California

Berkeley, CA 94720

STEVEN KeRCKHOFF

Stanford University

Stanford, CA 94305

\author{
ROBION KIRBY \\ University of California \\ Berkeley, CA 94720 \\ C. C. MOORE \\ University of California \\ Berkeley, CA 94720 \\ HAROLD STARK \\ University of California, San Diego \\ La Jolla, CA 92093
}

\section{ASSOCIATE EDITORS}
R. ARENS
E. F. BECKENBACH
B. H. NEUMANN
F. WOLF
K. YoshidA (1906-1982)

\section{SUPPORTING INSTITUTIONS}

UNIVERSITY OF ARIZONA

UNIVERSITY OF BRITISH COLUMBIA

CALIFORNIA INSTITUTE OF TECHNOLOGY

UNIVERSITY OF CALIFORNIA

MONTANA STATE UNIVERSITY

UNIVERSITY OF NEVADA, RENO

NEW MEXICO STATE UNIVERSITY

OREGON STATE UNIVERSITY
UNIVERSITY OF OREGON

UNIVERSITY OF SOUTHERN CALIFORNIA

STANFORD UNIVERSITY

UNIVERSITY OF HAWAII

UNIVERSITY OF TOKYO

UNIVERSITY OF UTAH

WASHINGTON STATE UNIVERSITY

UNIVERSITY OF WASHINGTON 


\section{Pacific Journal of Mathematics}

Vol. 140, No. $1 \quad$ September, 1989

Michel Brestovski, Algebraic independence of solutions of differential

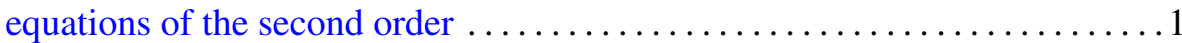

Bohumil Cenkl, Cohomology operations from higher products in the de

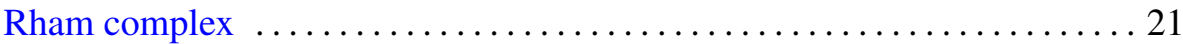

Gustavo Corach and Daniel Suarez, Generalized rational convexity in

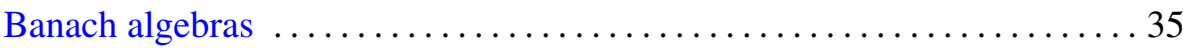

Keresztély Corrádi and Sándor Szabó, A new proof of Rédei’s theorem . . . 53

Steven R. Costenoble and Stefan Waner, Equivariant orientations and

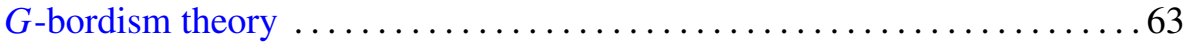

Angel Granja, Apéry basis and polar invariants of plane curve

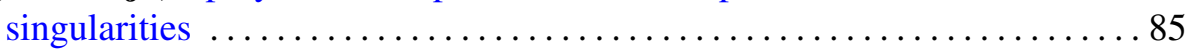

Young Soo Jo, Isometries of tridiagonal algebras .................. 97

Ronald Leslie Lipsman, Harmonic analysis on exponential solvable homogeneous spaces: the algebraic or symmetric cases $\ldots \ldots \ldots \ldots \ldots 117$

Erich Miersemann, On the behaviour of capillaries at a corner 149

Marian Nowak, On the finest Lebesgue topology on the space of essentially

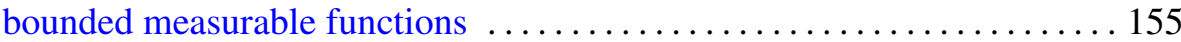

Pascal J. Thomas, Hardy interpolating sequences of hyperplanes ........ 163

H. Bevan Thompson, Differentiability properties of subfunctions for second order ordinary differential equations 